Macedonian Pharmaceutical Bulletin, 66 (Suppl 1) 181 - 182 (2020)

Online ISSN 1857 - 8969

UDC: $615.074: 543.544 .3$

DOI: 10.33320/maced.pharm.bull.2020.66.03.090

Short communication

\title{
Quantification using GC/ECD: challenges and pitfalls
}

\author{
Ana Poceva Panovska*, Jelena Acevska, Gabriela Petrovska Dimitrievska, \\ Katerina Brezovska, Natalija Nakov, Zoran Kavrakovski
}

Faculty of Pharmacy, Ss. Cyril and Methodius University, Majka Tereza 47, 1000 Skopje, North Macedonia

\section{Background}

Gas chromatography (GC) combined with electron capture detection (ECD) is commonly used techniques for analyzing organo-halogenated molecules which offers high selectivity and sensitivities at concentration ranges of parts-perbillion (ppb) or even parts-per-trillion ( $\mathrm{ppt}$ ). Comparing to the universal flame ionization detector (FID), an ECD can be $10-10^{3}$ times more sensitive depending on the analyte tested.

Despite the advantages, ECD is often described as a detector with limited dynamic linear range with linearity ranging between $10^{3}-10^{4}$ (Rood, 2007). Problems with the linearity are quite essential for quantifying an analyte because they define the range of the method within which the results are obtained accurately and precisely (Booij et al., 1998).

The aim of this review is to list some of possible reasons that might be involved in the non-linear response of the ECD that could often be source of ambiguous quantification, as well to address some of practical approaches to solve that.

\section{ECD related non-linearity}

The first point to inspect shouldn't be the detector itself, but the samples, especially if the onset of the non-linearity problem is unexpected. Inaccuracies in preparation, possible degradation of the substances, and evaporation of solvent and consequently variations in concentration are few of potential causes of non-linearity.

\footnotetext{
*anpo@ff.ukim.edu.mk
}

Any changes in the injection process also can alter the amount of sample entering the column and further significantly affect the linear response in the experiment. Inconsistent manual injection gives difference in the applied amount that usually leads to loss of repeatability and worsening of linear response. Adsorption of the analytes by the column or injector liner, may contribute to poor linear response since there is a reduction in the amount of compound that reaches the detector. In general, a compound at a lower concentration is more affected than the same compound at a higher concentration, and this often results in nonlinear calibration curves.

The detector is another potential source of nonlinear calibration curves. ECD is an ionization detector and its response is based upon the ability of molecules with certain functional groups to capture electrons generated by the radioactive source. The non-linearity characteristics of the ECD are often due to the physics of the response mechanism, since it is a "subtractive" detector. It means that maximum signal is obtained with no compounds in the detector by a steady background current that is formed by ions using the ionization source $\left({ }^{63} \mathrm{Ni}\right)$. The electronegative samples going through the detector, reduce the signal (by capturing electrons) versus adding signal in most other detectors, explaining the poor (linear) dynamic range of the ECD. (McNair and Miller, 2009)

Decrease in the linear range is one of the first signs of a contaminated ECD cell with high concentration of analyte. Due to contamination background current in the detector reduces, but the current reduction per analyte molecule remains 
constant which leads to non-linear response. Contaminated detectors usually give rise to negative 'dip' after each peak.

Another issue concerning the linearity is that ECD is a concentration type of detector. It measures concentration of the analyte in the carrier gas compared to other detectors (FID) that directly measure the absolute amount of analyte irrespective of the volume of the carrier gas. Due to this characteristic of ECD, quantitative data acquired at different flow rates will be affected. Therefore operation at constant flow may be necessary for quantitative analysis in programmed temperature GC. Additionally make-up gas should also be used. Lower rates of make-up gas will increase the sensitivity but it also increases the non-linearity. (McNair and Miller, 2009).

\section{Practical approach in solving the non-linearity}

When developing GC-ECD method for quantification purposes in laboratory few practical approaches should be consider and tested in order to check and solve potential non-linearity.

Check the linearity? Is the concentration of the analytes within the linearity range of the ECD?

Linear range and its end points have to be determined experimentally as they are often different for various compounds. If multiple point calibration curves are not generated, the loss of linear range may not be noticed until the errors in the quantitative results become quite severe. One of the approaches to alter the column/detector loadings is to increase the split flow but dilution of the too concentrated samples and re-injection is sometimes necessary.

\section{Are there obvious reasons for the nonlinear curve?}

Check sample concentration (they may fall outside the linear range of an ECD), injection technique, adjust the split flow, column flow and make-up flow. Look at the results of calibrations because sometimes the problem might be poor repeatability, not necessarily poor linearity. To avoid sample mistakes preparing additional set of samples or using another GC are the easiest methods to determine accountably of the prepared samples.

\section{Check for possible coelution analytes?}

In multicomponent analysis co-elution of components may contribute to non-linearity. Therefore the selectivity of the column for the compounds of interest must be checked. Column substitute might be a solution.

Can the dependence between the concentration and the response be explained by another mathematical model?

The log transformation can decrease the variability of data and make data conform more closely to the normal distribution. However, the results of standard statistical tests performed on logtransformed data are often not relevant for the original, non-transformed data, so this approach must be applied very cautiously. Instead newer analytic methods that are not dependent on the distribution the data (Feng et al., 2014) should be used.

\section{Conclusion}

Lack of linearity is often source of ambiguous quantification using ECD. There are some possible experimental hints that need to be determined in order for the linearity to be achieved. If the linearity remains questionable, the method should only be intended for semi-quantitative determination of the analyte concentration. Where possible, an alternative detector with broader linearity range and suitable sensitivity could be used.

\section{References}

Booij, K., Hillebrand M.T.J., van Weerlee E.M., 1998. Calibration of the electron-capture detector for the determination of polychlorinated biphenyls. Analyst $123,415-420$.

Feng, C., Wang, H., Lu, N., Chen, T., He, H., Lu, Y., Tu, X.M., 2014. Log-transformation and its implications for data analysis. Shanghai Archives of Psychiatry 26(2), $105-109$.

McNair, H.M., Miller, J.M., 2009. Basic gas chromatography. Hoboken, N.J.: John Wiley \& Sons.

Rood, D., 2007. The troubleshooting and maintenance guide for gas chromatographers. Wiley-VCH Verlag $\mathrm{GmbH} \& \mathrm{Co} . \mathrm{KGaA}$.

Ritsema, R., Donard, O.F.X., 2000. Organometallic compound determination in the environment by hyphenated techniques, in: BarcelÓ, D. (Eds.), Sample handling and trace analysis of pollutants. Science Direct, pp. 1003-1073.

Maced. Pharm. Bull. 66 (Suppl 1) 181 - 182 (2020) 